\title{
Current Management of Scaphoid Nonunion Based on the Biomechanical Study
}

\author{
Kunihiro Oka, MD, $\mathrm{PhD}^{1}$ Hisao Moritomo, MD, $\mathrm{PhD}^{2}$ \\ ${ }^{1}$ Health and Counseling Center, Department of Orthopaedic Surgery, \\ Osaka University Graduate School of Medicine, Osaka, Japan \\ 2 Department of Physical Therapy, Osaka Yukioka College of Health \\ Science, Osaka, Japan \\ Address for correspondence Hisao Moritomo, MD, PhD, Department \\ of Physical Therapy, Osaka Yukioka College of Health Science, \\ 1-1-41 Sojiji, Ibaraki-shi, Osaka 567-0801, Japan \\ (e-mail: hisao-moritomo@yukioka-u.ac.jp).
}

J Wrist Surg 2018;7:94-100.

\begin{abstract}
\section{Keywords}

- scaphoid nonunion

- natural history

- carpal collapse

- three-dimensional analysis

Scaphoid nonunion causes abnormal wrist kinematics and typically leads to carpal collapse and subsequent degenerative arthritis of the wrist. However, the natural history, including carpal collapse and degenerative arthritis of scaphoid nonunion, may vary at different fracture locations. This article reviews recent biomechanical studies related to the natural history of scaphoid nonunion. In the distal-type fractures (type B2 in Herbert classification), where the fracture located distal to the scaphoid apex, the proximal scaphoid fragment and lunate, which are connected through the dorsal scapholunate interosseous ligament (DSLIL) and dorsal intercarpal ligament (DIC), extend together, and the distal fragment of the scaphoid flexes individually. Therefore, untreated type B2 fractures normally show the humpback deformity, resulting in dorsal intercalated segment instability deformity relatively earlier after the injury. In the proximal-type fractures (type B1), where the fracture is located proximal to the scaphoid apex, the connection between the distal fragment and lunate is preserved through the DSLIL and DIC so that the scaphoid-lunate complex remains stable and the carpal collapse is less severe than that in distal-type fractures. The fracture location relative to the apex of the dorsal scaphoid ridge is a reliable landmark in the determination of the natural history of scaphoid nonunion.
\end{abstract}

Scaphoid fractures are the most common fractures in carpal bones, accounting for 80 to $90 \%$ of all carpal fractures. ${ }^{1,2}$ Their frequency of occurrence is 12.4 of 100,000 fractures per year, and these fractures most commonly occur in young males. ${ }^{3}$ The nonunion rate after scaphoid fracture is relatively high. Even when scaphoid fractures are adequately treated using casts, the reported nonunion rate has been as high as approximately $10 \%{ }^{4}$ Scaphoid nonunion results in abnormal wrist kinematics and typically leads to carpal collapse and a subsequent degenerative arthritis of the wrist. $^{5}$ Most scaphoid nonunions are symptomatic and show scaphoid humpback and dorsal intercalated segment instability (DISI) deformities. However, it is true that some cases are less symptomatic with gradual carpal collapse; these cases do not receive the required medical attention for

received

December 21, 2017

accepted

February 8, 2018

published online

March 14, 2018 a prolonged period. ${ }^{6}$ In recent years, the patterns of carpal collapse in scaphoid nonunions have been investigated in clinical examinations and cadaveric biomechanical studies based on the fracture type. ${ }^{7-14}$ The natural history, including carpal collapse and degenerative arthritis, of scaphoid nonunion may vary because of the fracture locations. ${ }^{15}$ The purpose of this article was to review recent studies on the biomechanics of scaphoid nonunion to demonstrate different natural histories of scaphoid nonunion.

\section{Classification}

In 1984, Herbert classified scaphoid fractures into stable fractures, unstable fractures, delayed unions, and nonunions. ${ }^{8}$ Herbert type B fractures are unstable acute complete fractures

Copyright $\odot 2018$ by Thieme Medical Publishers, Inc., 333 Seventh Avenue, New York, NY 10001, USA Tel: +1(212) 584-4662.
DOI https://doi.org/ 10.1055/s-0038-1637739. ISSN 2163-3916. 


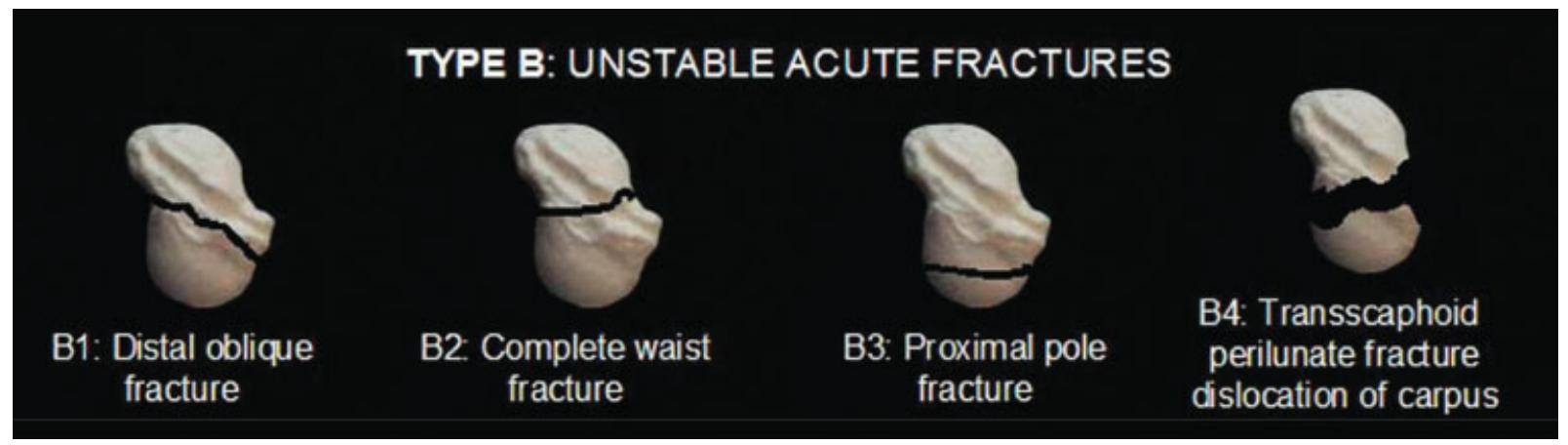

Fig. 1 Type B in the Herbert classification of scaphoid fractures. (Reproduced with permission from Haisman JM, Rohde RS, Weiland AJ. Acute fractures of the scaphoid. J Bone Joint Surg Am 2006;88:2750-2758.)

that are classified into four subtypes on the basis of the fracture location. In 2006, a depiction of more definite fracture locations of the four subtypes using a three-dimensional (3D) scaphoid model was published in The Journal of Bone and Joint Surgery $^{9}$ ( - Fig. 1). The fracture line in type B1 obliquely runs from the mid-third of the volar aspect to the proximal third of the dorsal aspect, type B2 fracture is a transverse complete scaphoid waist fracture, type B3 fracture is a proximal pole fracture, and type B4 fracture is a transscaphoid perilunate fracture dislocation.

Nakamura et al have reported two different patterns of displacement, namely, volar and dorsal, in scaphoid nonunion. ${ }^{15}$ Distal scaphoid waist fractures usually develop in a volar-type pattern and are accompanied by DISI and humpback deformity of the scaphoid. In contrast, proximal scaphoid waist and pole fractures frequently develop in a dorsal-type pattern and are accompanied by a minor displacement of the scaphoid and carpal bones. Moritomo et al have suggested that both volar- and dorsal-type displacement patterns are specifically related to the fracture location with respect to the apex of the dorsal scaphoid ridge. ${ }^{10}$ They revealed that the fracture line of scaphoid nonunion in volar-type displacement is distal to the scaphoid apex (distal type) and that of scaphoid non- union in dorsal-type displacement is proximal to the scaphoid apex (proximal type) ( Fig. 2A, B). Distal and proximal types in their classification have been suggested to correspond to types B2 and B1, respectively, in the Herbert classification.

\section{Mechanism of Carpal Deformity}

Established scaphoid nonunion typically leads to carpal collapse and osteoarthritis of the wrist, known as scaphoid nonunion advanced collapse (SNAC). ${ }^{5}$ Several studies using 3D computed tomography (CT) have reported that wrist symptoms, the natural history of carpal collapse and rapidity of deformity, and the location of osteoarthritis depend on the fracture location with respect to the scaphoid apex. ${ }^{6,8,10-12,14,16}$ The mechanisms of the development of different deformity patterns based on the scaphoid apex are explained as follows (-Fig. 3A-E). As per classic anatomical and biomechanical studies on the normal wrist, the scaphoid is naturally subject to flexional load from the trapezium, trapezoid, and capitate through axial forces due to its anatomical shape. ${ }^{17-20}$ Conversely, the triquetrum is subject to extensional load from the hamate. The flexion moment sustained by the scaphoid is constrained by the extension moment experienced by the

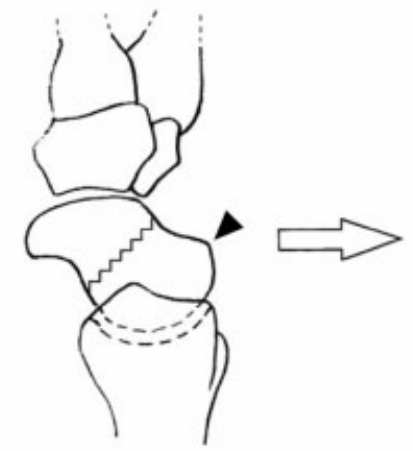

A

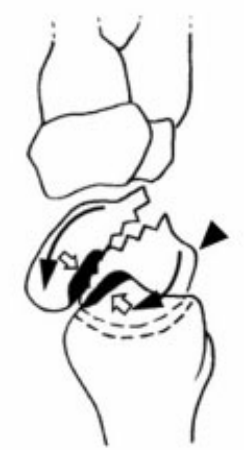

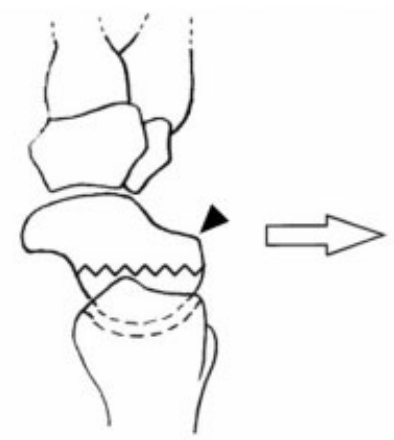

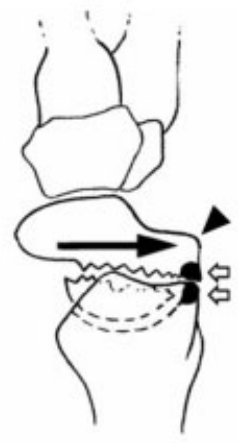

B

Fig. 2 Black triangles indicate the apex of the dorsal scaphoid ridge. (A) The volar type of scaphoid nonunion, as seen from the lateral view, showing the direction of fracture displacement (solid arrows) and the inferred contact area between the distal fragment of the scaphoid and the radius (open arrows). (B) The dorsal type of scaphoid nonunion, as seen from the lateral view, showing the direction of fracture displacement (solid arrow) and the inferred contact area between the distal fragment of the scaphoid and the radius (open arrows). (Reproduced with permission from Moritomo H, Viegas SF, Elder KW, Nakamura K, Dasilva MF, Boyd NL, Patterson RM. Scaphoid nonunions: a 3-dimensional analysis of patterns of deformity. J Hand Surg Am 2000;25:520-528.) 


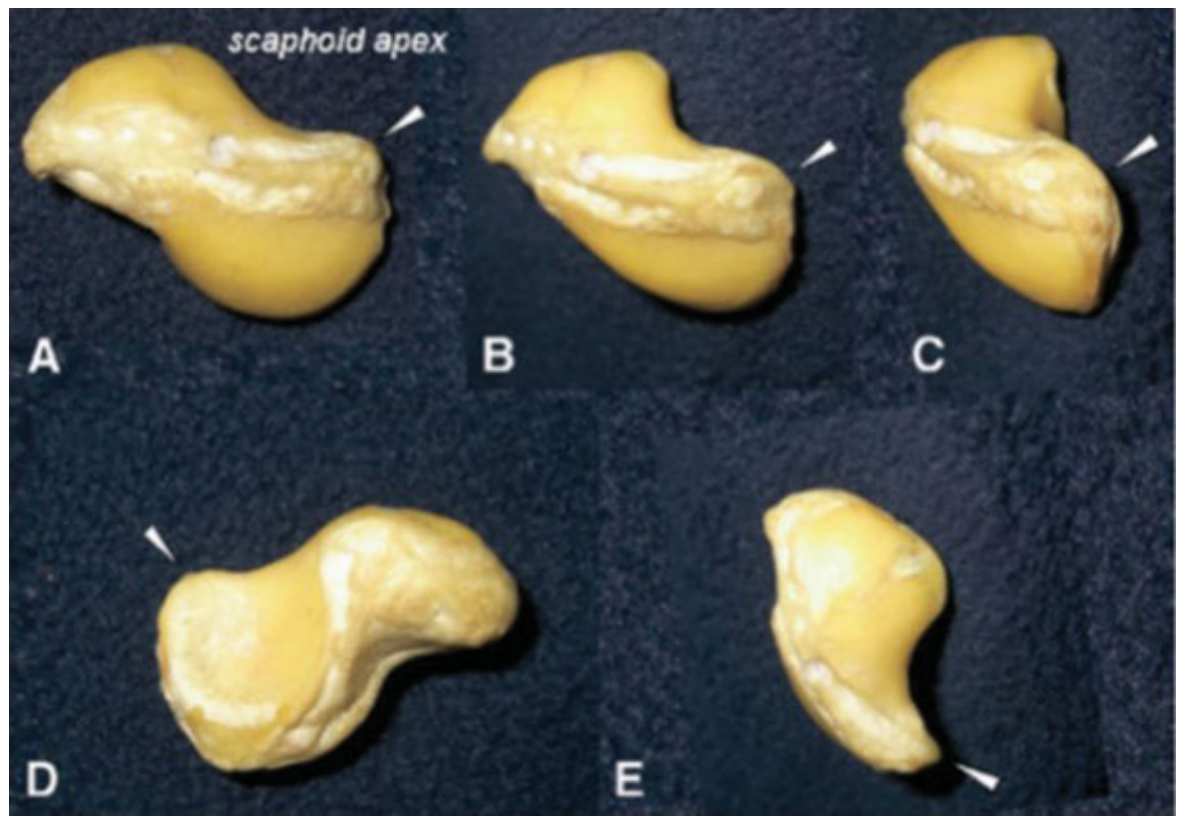

Fig. 3 The scaphoid apex is the most dorsal and ulnar nonarticulating part of the scaphoid. The scaphoid of a right cadaver wrist seen from the (A) radial, (B) radiodorsal, (C) dorsal, (D) ulnar, and (E) distal side. The location of the scaphoid apex on the dorsal ridge is indicated by an arrow head. (Reproduced with permission from Moritomo H, Murase T, Oka K, Tanaka H, Yoshikawa H, Sugamoto K. Relationship between the fracture location and the kinematic pattern in scaphoid nonunion. J Hand Surg Am 2008;33:1459-1468.)

triquetrum, and a stable equilibrium is achieved. Garcia-Elias compared this equilibrium to a spring with two arms (scaphoid and triquetrum) distally prolonged in divergent directions. ${ }^{17}$ dorsal scapholunate interosseous ligament (DSLIL) and the proximal fiber of the dorsal intercarpal ligament (DIC), which connect the scaphoid and lunate and are attached to the

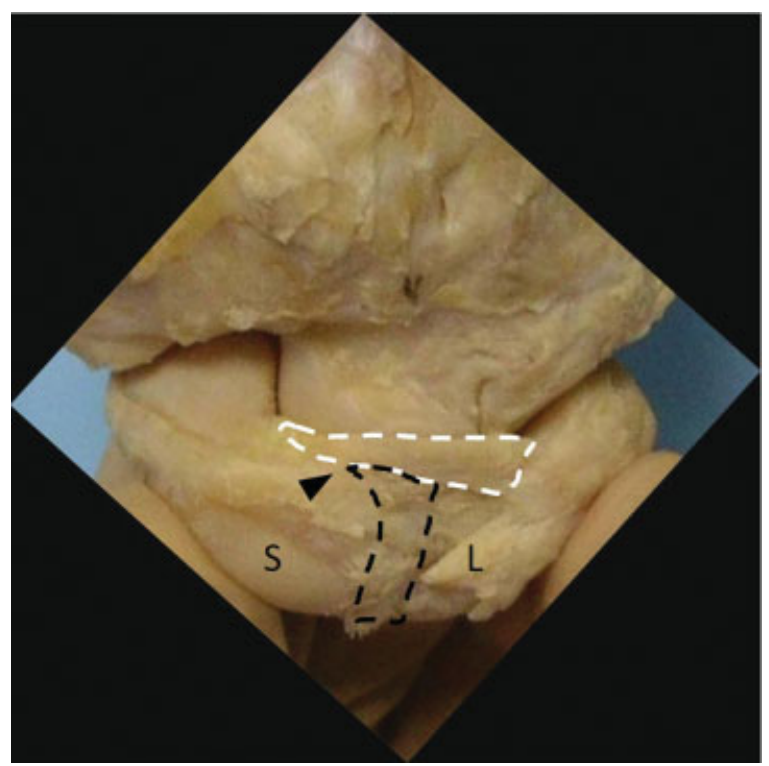

Fig. 4 Dorsal view of the wrist. Areas surrounded by white and black dotted lines indicate the proximal fibers of the dorsal intercarpal ligament (DIC) and the dorsal scapholunate interosseous ligament (DSLIL), respectively. The proximal fibers of the DIC are attached from the scaphoid (S) apex (black triangle) to the dorsal lunate (L), and the DSLIL connects the proximal portion of the scaphoid to the apex and lunate. scaphoid apex, play an important role in preventing carpal collapse (- Fig. 4). ${ }^{17}$ In distal-type fractures (type B2), where the fracture is located distal to the scaphoid apex, the scaphoidlunate complex is separated into two segments, namely, the lunate and proximal fragment of the scaphoid and the distal fragment of the scaphoid at the fracture site. The proximal scaphoid fragment and lunate, which are connected through the DSLIL and DIC, extend together, and the distal fragment of the scaphoid flexes individually. ${ }^{10,11,21}$ Therefore, untreated distal-type fractures (type B2) result in humpback and DISI deformities and are accompanied by large bone defects on the volar side, relatively early after injury. ${ }^{5,8,9,22-25}$ The dorsal translation and flexion of the distal fragment caused by DISI deformity may create an impingement between the distal fragment and radial styloid, leading to osteophyte formation at the radial styloid (-Fig. 5) ${ }^{11}$ Degenerative change at the radial styloid does not always occur in the distal type. ${ }^{9,26}$ In the distal extra-articular type, the distal fragment of the scaphoid does not impinge on the radial styloid even when humpback deformity is developed because the fracture line is extraarticular to the radioscaphoid joint. ${ }^{12}$ In the distal intra-articular type, flexion and dorsal translation of the distal fragment cause a conflict between the distal fragment and the radial styloid, leading to a degenerative change at the radial styloid.

In proximal-type fractures (type B1), where the fracture is located proximal to the scaphoid apex, the connection between the distal fragment and lunate is preserved through the DSLIL and DIC so that the scaphoid-lunate complex remains stable and carpal collapse is less severe than that in distal-type fractures. ${ }^{10,17}$ While proximal-type fractures have a degree of carpal stability, minor movements at the nonunion affect the radioscaphoid joint over time. Chronic 


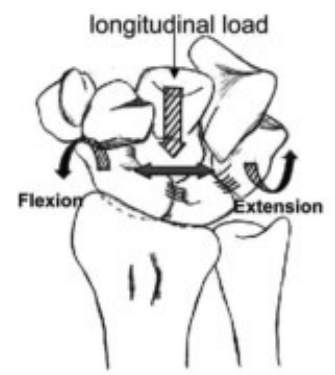

A

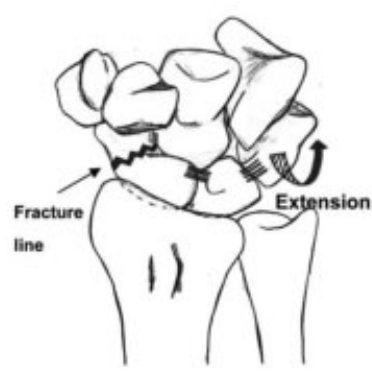

B

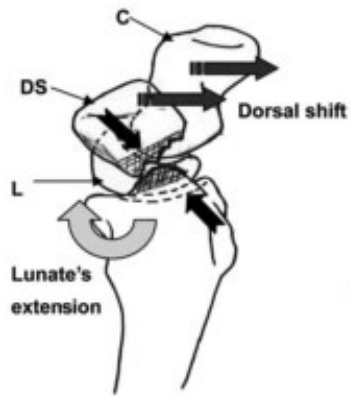

$\mathrm{C}$

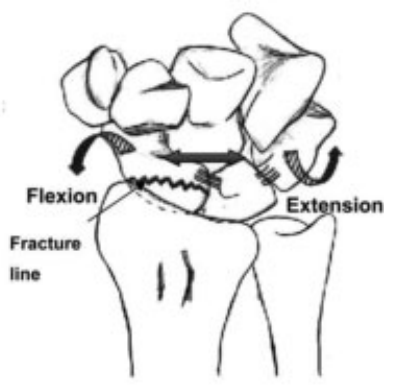

D

Fig. 5 (A) Force of the scaphoid rotating into flexion and pronation counters the force of the triquetrum rotating into extension and supination. With distal fractures, the link to the proximal row is broken at the fracture site. (B) The proximal fragment of the scaphoid and lunate, and the triquetrum extend and supinate. (C) The capitate and the distal fragment translate in the dorsal direction due to the effect of the extension of the lunate. (D) With proximal fractures, the link to the proximal row survives and the proximal row remains stable. (Reproduced with permission from Oka K, Moritomo H, Murase T, Goto A, Sugamoto K, Yoshikawa H. Patterns of carpal deformity in scaphoid nonunion: a 3-dimensional and quantitative analysis. J Hand Surg Am 2005;30:1136-1144.)

proximal-type fractures generate widespread osteophytes at the dorsal scaphoid fossa on the radius and at the dorsal scaphoid ridge on the scaphoid due to the impingement of the large distal scaphoid fragment and radius (-Fig. 6). ${ }^{12}$

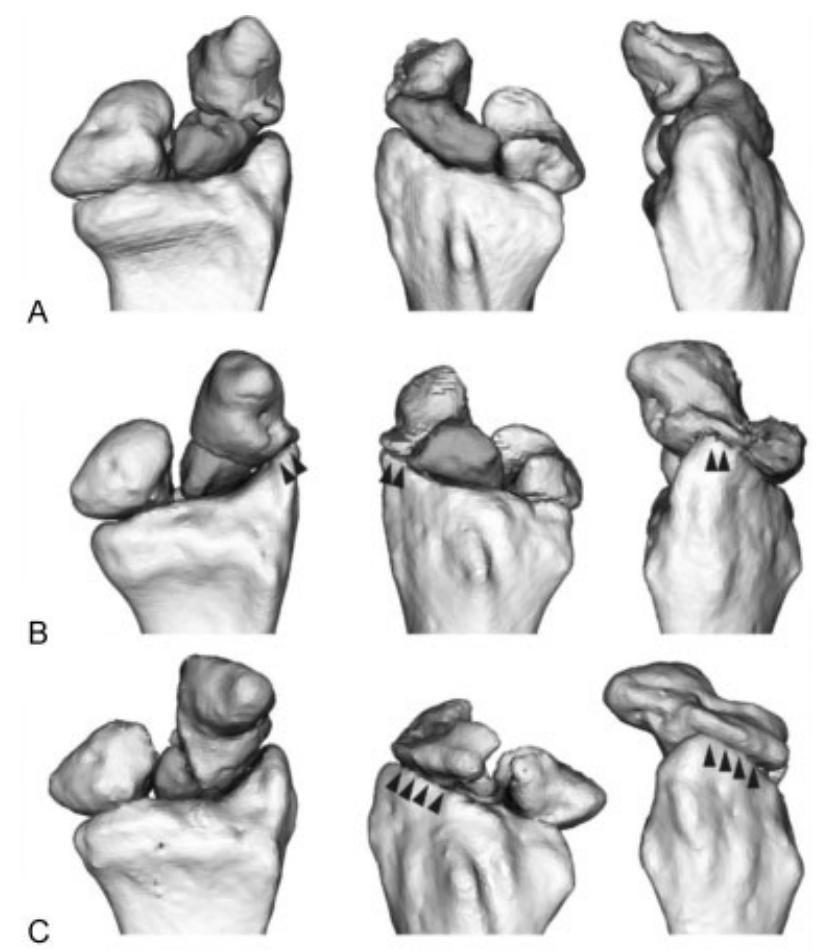

Fig. 6 Three-dimensional models of the radius, lunate, and fragments of the scaphoid. (A) Distal extra-articular type. There were few degenerative changes in the radioscaphoid joint. (B) Distal intra-articular type. The distal fragment impinges on the radial styloid (arrow heads). Pointing of the radial styloid was present. (C) Proximal type. The distal fragment impinges on the dorsal scaphoid fossa of the radius (arrow heads). Osteophyte formation was seen from the styloid process to the dorsal aspect of the scaphoid fossa of the radius. (Reproduced with permission from Oura K, Moritomo $\mathrm{H}$, Kataoka T, Oka K, Murase T, Sugamoto K, Yoshikawa H. Three-dimensional analysis of osteophyte formation on distal radius following scaphoid nonunion. J Orthop Sci 2017;22:50-55.)

\section{Kinematics}

The scaphoid radially connects the intercalated segment and the distal row. The distal scaphoid pole is connected to the trapezium through the scaphotrapezial ligament, and the palmar radioscaphoid capitate and scaphoid capitate ligaments are secondary stabilizers between the scaphoid and distal row. ${ }^{27,28}$ The scaphoid apex and proximal pole are linked with the lunate through the scapholunate interosseous ligament (SLIL). These ligamentous and osseous structures of the scaphoid provide a considerable degree of stability to carpal bones. Scaphoid fractures may change the carpal kinematics through the pathological interfragmentary motion of the scaphoid. In a cadaveric study on scaphoid waist fractures, interfragmentary motion was mainly observed in the flexional motion of the distal fragment and in the limited motion of the proximal fragment during wrist flexion. ${ }^{19}$

Recently, pathological kinematics in scaphoid nonunion has been elucidated using noninvasive in vivo 3D kinematic studies. ${ }^{8,29}$ Leventhal et al investigated the interfragmentary motion of scaphoid nonunion in six patients ${ }^{29}$ and reported that distal fragment motion during wrist flexion and extension is similar to that in the normal scaphoid. In contrast, the extension of the proximal fragment and lunate is significantly decreased by 38 and $40 \%$, respectively. The interfragmentary motion of scaphoid nonunion was found to be onethird than that of total wrist motion. They suggested that abnormal carpal motion was caused because of the failure of a fundamental link between the proximal and distal carpal rows at the fracture site. Moritomo et al have revealed that interfragmentary motions of scaphoid nonunion show two patterns, namely, mobile and stable types, based on the fracture location; type B2 fractures (where the dorsal portion of the fracture was distal to the scaphoid apex) showed a mobile-type pattern in which the distal fragment was unstable relative to the proximal fragment. ${ }^{8}$ Osseous break of the scaphoid resulted in the failure of a coordinated motion between the intercalated segment and the distal 


\section{B1 fracture}

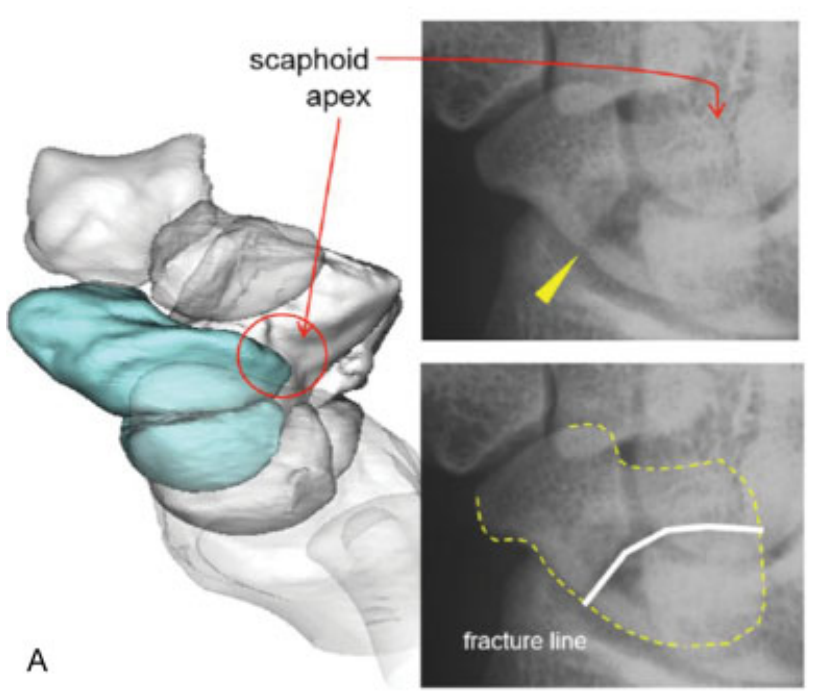

B2 fracture

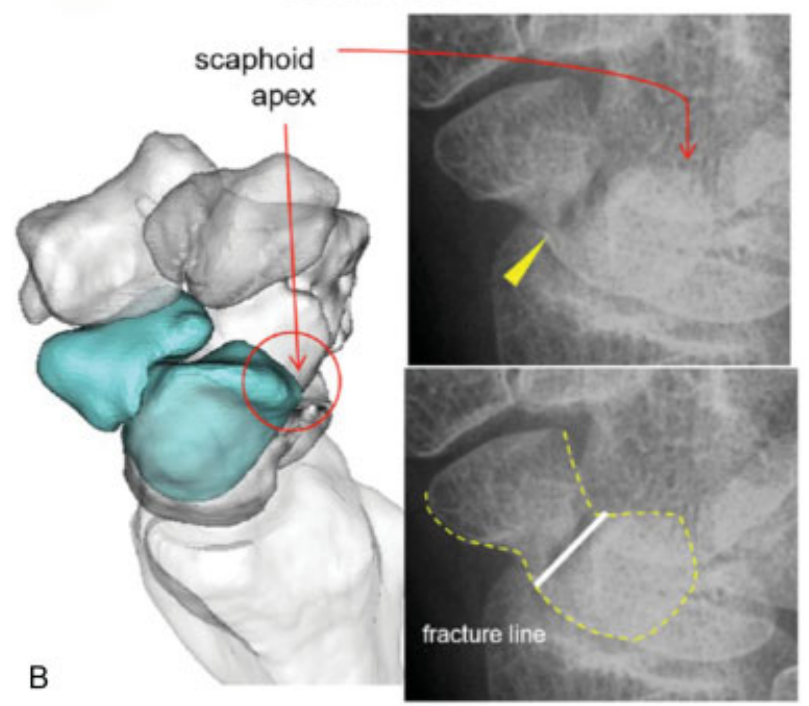

Fig. 7 Three-dimensional (3D) images and oblique radiographs of (A) B1 and (B) B2 scaphoid nonunions. Although the relationship between the fracture line and scaphoid apex differed on the 3D images, both fracture lines appear similar on radiographs (arrowheads). (Reproduced with permission from Moritomo H. Radiographic clues for determining carpal instability and treatment protocol for scaphoid fractures. J Orthop Sci 2014;19:379-383.)

row. The distal fragment moved along with the distal row, whereas the proximal fragment moved along with the proximal row. The distal fragment flexed 7 degrees and extended 24 degrees relative to the proximal fragment with wrist flexion and extension, manifesting as a "bookopening" motion. Furthermore, they found that the distal scaphoid fragment impinged on the radial styloid in wrist radial deviation. Type B1 fractures (where the dorsal portion of the fracture was proximal to the scaphoid apex) showed a stable-type pattern in which the interfragmentary motion was minor during wrist motion. The distal fragment moved $<10$ degrees relative to the proximal fragment during wrist flexion and extension because the distal fragment was stabilized to the lunate through the proximal fibers of the DIC and DSLIL. Carpal kinematics during wrist motion was relatively preserved, and carpal collapse was not obvious.

Changes in carpal kinematics in scaphoid nonunion based on the fracture location (distal or proximal to the scaphoid apex) were also verified using fresh-frozen cadaver wrists. ${ }^{14}$ Fracture lines distal or proximal to the scaphoid apex were created in six and five wrists, respectively. The study validated wrist motion in these two types of scaphoid fracture models. The interfragmentary motion was significantly greater in the distal type (type B2) than in the proximal type (type B1). The distal fragment flexed and pronated relative to the proximal fragment significantly more in the distal type than in the proximal type during wrist motion, flexion-extension, radioulnar deviation, and dartthrow motion. Moreover, the study found that the greatest interfragmentary motion was typically seen during a flexionextension axis of movement and that its motion was remarkable during wrist flexion. Therefore, we surmise that carpal collapse and instability following scaphoid nonunion are strongly related to whether the fracture line can pass distally or proximally to the scaphoid apex where the DSLIL and DIC attach.

\section{Diagnosis and Treatment}

The principal goals of the treatment of scaphoid nonunion include achieving union, correcting the scaphoid deformity, and restoring the carpal alignment to prevent a degenerative change of the wrist. As mentioned previously, the pattern of carpal collapse and location of osteophyte generation are quite different between the proximal (type B1) and distal (type B2) types. Therefore, it is important to preoperatively distinguish whether the fracture is located proximal or distal to the scaphoid apex. Three-dimensional CT is a useful imaging modality to diagnose the fracture type and to formulate the best strategy for the treatment of scaphoid nonunion. Plain radiographs with careful observation can also detect whether the fracture location is proximal or distal to the scaphoid apex (-Fig. 7). ${ }^{16,30}$ In a 45-degree pronated oblique view, the scaphoid apex can be observed overlapping the capitate head. Distal- or proximal-type fractures can be identified by identifying the fracture location relative to the scaphoid apex. In a posteroanterior radiograph with the wrist in the neutral position, obvious differences between the proximal- and distal-type fractures are observed. In proximal-type fractures, the fracture line is manifestly observed because the fracture line runs nearly horizontal to the wrist. Conversely, in distal-type fractures, the fracture line is obscure because it is vertical to the scaphoid long axis and oblique to the posteroanterior direction of the wrist. However, the diagnosis of fracture types using plain radiographs has limited accuracy; therefore, evaluation using 3D CT can be recommended.

Distal-type fractures (type B2) are normally subjects to open reduction and internal fixation as early as possible because the DISI deformity rapidly may progress, resulting in subsequent SNAC deformity. When distal-type scaphoid nonunion is treated with the surgery, the volar approach is 


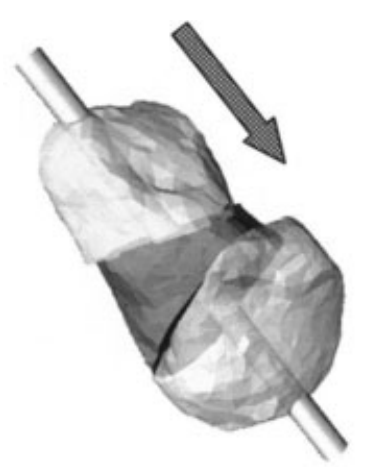

A

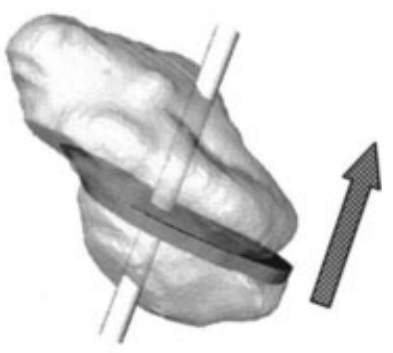

B

Fig. 8 Pattern diagrams of bone grafting and screwing. (A) Distal type: wedge-shaped bone grafting and screwing from a volar approach is easier. (B) Proximal type: grafting of bone chips from the dorsal side is recommended. Screw insertion from the dorsal side allows it to penetrate the fracture site vertically. (Reproduced with permission from Oka K, Murase T, Moritomo H, Sugamoto K, Yoshikawa $\mathrm{H}$. Patterns of bone defect in scaphoid nonunion: a 3-dimensional and quantitative analysis. J Hand Surg Am 2005;30:359-365.)

preferable because of the presence of large bone defects on the volar side. Grafting a wedge-shaped bone harvested from the iliac crest can correct the humpback deformity and restore the scaphoid length. Screws can be conveniently inserted through the scaphoid tuberosity to confirm the bone graft position. While open reduction with internal fixation is the first choice of management for proximal-type fractures (B1), conservative treatment can be considered an optional therapy in elderly patients or in those who may not prefer surgical treatment due to economic or social reasons because these fractures are less symptomatic. When the proximal-type fractures are treated with open reduction and internal fixation, a dorsal approach is recommended. Osteophytes developed on the dorsal scaphoid fossa of the radius and the dorsal scaphoid ridge can be observed from the dorsal side in a wrist flexional position. After the removal of these dorsal osteophytes, the nonunion is approached with a curette, and a small amount of cancellous bone graft is filled into the nonunion site. Moreover, screw insertion from the dorsal side allows vertical fixation of the fracture site (-Fig. 8). ${ }^{6}$

The DISI deformity and carpal instability occasionally develop in proximal-type fractures when SLIL is torn or loosened. When the fracture line runs close to the scaphoid apex in proximal fractures, the remaining SLIL still attached to the distal fragment may possibly get damaged. It is essential not only to diagnose the possible carpal instability by discerning fracture types but also to pay careful attention to the possibility of a latent SLIL tear. If dysfunction of the SLIL is suspected with a separation between the scaphoid apex and the dorsal lunate horn, then additional dynamic evaluation, fluoroscopy, and dynamic CT are necessary.

\section{Conclusion}

This review article summarizes the current consensus on the natural history of scaphoid nonunion based on 3D analysis. Although problems regarding the concomitant presence of carpal ligament injury, perilunate dislocation, or multiple fragmentations of scaphoid are yet to be solved, the fracture location relative to the apex of the dorsal scaphoid ridge is a reliable landmark to determine the natural history of scaphoid nonunion. Therefore, the evaluation of scaphoid nonunion using 3D CT is recommended to determine the fracture type, which helps to formulate the best strategy for management.

\section{Funding}

The authors were supported by JSPS KAKENHI (grant number JP 15K10442).

\section{Conflict of Interest \\ None.}

\section{References}

1 Brogan DM, Moran SL, Shin AY. Outcomes of open reduction and internal fixation of acute proximal pole scaphoid fractures. Hand (NY) 2015;10(02):227-232

2 Leslie IJ, Dickson RA. The fractured carpal scaphoid. Natural history and factors influencing outcome. J Bone Joint Surg $\mathrm{Br}$ 1981;63-B(02):225-230

3 Garala K, Taub NA, Dias JJ. The epidemiology of fractures of the scaphoid: impact of age, gender, deprivation and seasonality. Bone Joint J 2016;98-B(05):654-659

4 Dias JJ, Brenkel IJ, Finlay DB. Patterns of union in fractures of the waist of the scaphoid. J Bone Joint Surg Br 1989;71(02):307-310

5 Mack GR, Bosse MJ, Gelberman RH, Yu E. The natural history of scaphoid non-union. J Bone Joint Surg Am 1984;66(04):504-509

6 Oka K, Murase T, Moritomo H, Goto A, Sugamoto K, Yoshikawa H. Patterns of bone defect in scaphoid nonunion: a 3-dimensional and quantitative analysis. J Hand Surg Am 2005;30(02):359-365

7 Gehrmann S, Roeger T, Kaufmann R, Schaedle A, Lögters T, Windolf $\mathrm{J}$. Wrist motion analysis in scaphoid nonunion. Eur $\mathrm{J}$ Trauma Emerg Surg 2016;42(01):11-14

8 Moritomo H, Murase T, Oka K, Tanaka H, Yoshikawa H, Sugamoto K. Relationship between the fracture location and the kinematic pattern in scaphoid nonunion. J Hand Surg Am 2008;33(09):1459-1468

9 Moritomo H, Tada K, Yoshida T, Masatomi T. The relationship between the site of nonunion of the scaphoid and scaphoid nonunion advanced collapse (SNAC). J Bone Joint Surg Br 1999;81(05):871-876

10 Moritomo H, Viegas SF, Elder KW, et al. Scaphoid nonunions: a 3dimensional analysis of patterns of deformity. J Hand Surg Am 2000;25(03):520-528

11 Oka K, Moritomo H, Murase T, Goto A, Sugamoto K, Yoshikawa H. Patterns of carpal deformity in scaphoid nonunion: a 3-dimensional and quantitative analysis. J Hand Surg Am 2005;30(06):1136-1144

12 Oura K, Moritomo H, Kataoka T, et al. Three-dimensional analysis of osteophyte formation on distal radius following scaphoid nonunion. J Orthop Sci 2017;22(01):50-55

13 Schweizer A, Fürnstahl P, Nagy L. Three-dimensional computed tomographic analysis of 11 scaphoid waist nonunions. J Hand Surg Am 2012;37(06):1151-1158

14 Werner FW, St-Amand H, Moritomo H, Sutton LG, Short WH. The effect of scaphoid fracture site on scaphoid instability patterns. J Wrist Surg 2016;5(01):47-51

15 Nakamura R, Imaeda T, Horii E, Miura T, Hayakawa N. Analysis of scaphoid fracture displacement by three-dimensional computed tomography. J Hand Surg Am 1991;16(03):485-492

16 Moritomo H. Radiographic clues for determining carpal instability and treatment protocol for scaphoid fractures. J Orthop Sci 2014;19(03):379-383

17 Garcia-Elias M. Kinetic analysis of carpal stability during grip. Hand Clin 1997;13(01):151-158 
18 Kauer JM. Functional anatomy of the wrist. Clin Orthop Relat Res 1980;(149):9-20

19 Smith DK, Cooney WP III, An KN, Linscheid RL, Chao EY. The effects of simulated unstable scaphoid fractures on carpal motion. J Hand Surg Am 1989;14(2 Pt 1):283-291

20 Weber ER. Biomechanical implications of scaphoid waist fractures. Clin Orthop Relat Res 1980;(149):83-89

21 Garcia-Elias M, Lluch A. Partial excision of scaphoid: is it ever indicated? Hand Clin 2001;17(04):687-695

22 Hidaka Y, Nakamura R. Progressive patterns of degenerative arthritis in scaphoid nonunion demonstrated by three-dimensional computed tomography. J Hand Surg Br 1998;23(06):765-770

23 Inoue G, Sakuma M. The natural history of scaphoid non-union. Radiographical and clinical analysis in 102 cases. Arch Orthop Trauma Surg 1996;115(01):1-4

24 Kozin SH. Incidence, mechanism, and natural history of scaphoid fractures. Hand Clin 2001;17(04):515-524
25 Ruby LK, Stinson J, Belsky MR. The natural history of scaphoid non-union. A review of fifty-five cases. J Bone Joint Surg Am 1985; 67(03):428-432

26 Vender MI, Watson HK, Wiener BD, Black DM. Degenerative change in symptomatic scaphoid nonunion. J Hand Surg Am 1987;12(04):514-519

27 Lee DJ, Elfar JC. Carpal ligament injuries, pathomechanics, and classification. Hand Clin 2015;31(03):389-398

28 Short WH, Werner FW, Green JK, Sutton LG, Brutus JP. Biomechanical evaluation of the ligamentous stabilizers of the scaphoid and lunate: part III. J Hand Surg Am 2007;32(03):297-309

29 Leventhal EL, Wolfe SW, Moore DC, Akelman E, Weiss AP, Crisco JJ. Interfragmentary motion in patients with scaphoid nonunion. J Hand Surg Am 2008;33(07):1108-1115

30 Inagaki H, Nakamura R, Horii E, Nakao E, Tatebe M. Differences in radiographic findings between scaphoid fracture patterns. Hand Surg 2004;9(02):197-202 